\title{
NOUVELLE
}

\section{Altération de l'autophagie chez les patients atteints de maladie de Crohn}

\section{Une porte ouverte pour des bactéries invasives?}

Anne-Lise Glasser, Pierre Lapaquette, Arlette Darfeuille-Michaud
Pathogénie Bactérienne Intestinale, JE2526 Université d'Auvergne, USC-INRA 2018, CBRV, 63000 Clermont-Ferrand, France.

a-lise.glasser@u-clermontl.fr

pierre.lapaquette@u-clermontl.fr arlette.darfeuille-michaud@u-clermontl.fr
> L'autophagie, du Grec «autos » (de soimême) et «phagos» (manger), est un processus biologique d'autocannibalisme qui conduit à la dégradation par le lysosome de constituants cellulaires (agrégats protéiques, organites endommagés ou surnuméraires). L'autophagie participe au maintien de l'homéostasie cellulaire et joue un rôle dans la différenciation, la survie et la mort cellulaires, dans la réponse à des stress environnementaux, et dans l'immunité innée et adaptative. Certains cancers, des maladies neurodégénératives (maladie de Huntington), des maladies inflammatoires chroni- ques de l'intestin (maladie de Crohn) ou certaines pathologies infectieuses pourraient avoir comme origine une altération de l'autophagie $(\rightarrow)$

$(\rightarrow)$ Voir les articles de P. Codogno et J. Puyal et al. p. 323 et p. 383 de ce numéro $[17,18]$.

\section{Mécanisme de l'autophagie}

L'autophagie est un processus faisant intervenir trois étapes: l'induction, l'élongation, et la maturation (Figure l). L'induction permet la mise en place d'une structure membranaire dite pré-autophagosomale. Elle est sous la dépendance

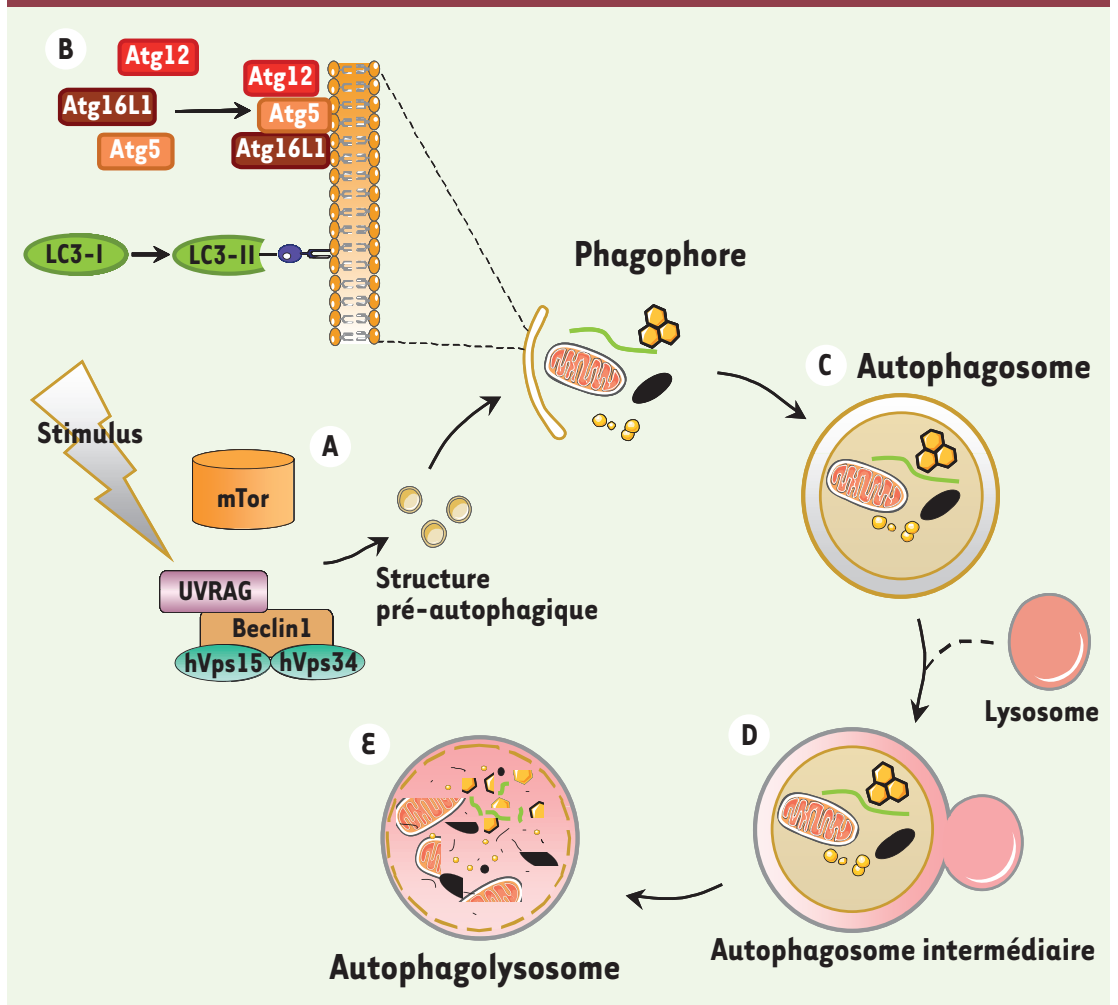

de la GTPase IRGM (immunity-related GTPase family, M) et de deux complexes protéiques, un premier qui fait intervenir la protéine mTor (mammalian target of rapamycin) et un second associant la protéine adaptatrice Beclin 1 [14] avec une PI3-kinase de classe III. L'élongation de cette structure conduit à la formation d'une membrane d'isolement, appelée phagophore, qui piège une portion de cytoplasme et capture les éléments à ingérer. Cet événement dépend de deux systèmes de conjugaison similaires à l'ubiquitinylation des protéines et dont les acteurs majeurs sont LC3 et le complexe trimérique Atg5, Atg12 et Atgl6 Ll (autophagy-related 16-likel) [15]. La localisation de ces complexes au niveau de la membrane d'isolement est indispensable pour la formation d'une

Figure 1. Schéma du processus autophagique. $\varepsilon n$ réponse à différents stimulus (carence en nutriments, hypoxie, modification de la balance énergétique AMP/ATP, présence de micro-organismes, production d'espèces oxygénées réactives, ADN endommagé), les complexes protéiques mTor et Beclinl induisent la formation d'une structure membranaire préautophagique ( $A$ ). Les 2 systèmes de conjugaison Atgl6Ll/Atg5/Atg12 et LC3 permettent la formation du phagophore par élongation de la membrane et séquestration d'éléments cytoplasmiques (protéines, organites ou bactéries) (B). La fermeture de la double membrane conduit à un autophagosome (C) qui fusionne avec des lysosomes (D) et conduit à la dégradation de la membrane interne et du contenu intra-vacuolaire $(\varepsilon)$. 
vacuole à double membrane, appelée autophagosome. Enfin, cet autophagosome fusionne avec des lysosomes pour constituer un autophagolysosome mature capable de dégrader son contenu (pour revue voir [1])

\section{Rôle de l'autophagie dans I'élimination de bactéries intracellulaires}

L'autophagie est une voie de dégradation de bactéries intracellulaires parallèle au trafic endocytaire classique. Elle permet de prendre en charge soit des bactéries libres dans le cytoplasme, soit des bactéries internalisées dans des vacuoles immatures ou endommagées. Les streptocoques du groupe $A$, grâce à la synthèse de streptolysine 0 , lysent la vacuole de phagocytose et se répliquent dans le cytoplasme de la cellule hôte. À ce stade, le processus d'autophagie séquestre ces bactéries et les élimine dans un autophagolysosome, comme l'ont montré des expériences réalisées avec des fibroblastes murins $\operatorname{atg} 5^{-/-}$incapables de réaliser un processus autophagique, dans lesquels les bactéries se multiplient beaucoup plus fortement que dans des cellules dont le gène $\operatorname{atg} 5$ est intact [2].

L'autophagie permet également la prise en charge de bactéries internalisées, comme Mycobacterium tuberculosis, qui sont capables de créer une niche de réplication dans une vacuole en bloquant la maturation du phagosome. Seule l'autophagie, induite en réponse à la privation des cellules en nutriments ou à une stimulation par l'interféron- $\gamma$, permet d'éliminer les mycobactéries internalisées [3]. La protéine IRGM joue un rôle clé dans cette défense antibactérienne qu'exerce l'autophagie comme cela a été montré dans des macrophages murins et humains dans lesquels la traduction de cette protéine était bloquée à l'aide d'ARN interférents [4].

Salmonella Typhimurium illustre une autre modalité de ciblage de bactéries intracellulaires par l'autophagie: cette bactérie pathogène à tropisme intesti- nal est capable également de modifier la maturation de son phagosome afin d'établir une niche de réplication. Cependant, une fraction des bactéries ainsi internalisées altère l'intégrité de la vacuole grâce à des effecteurs bactériens sécrétés via un système de sécrétion de type III, et se retrouvent libres dans le cytoplasme où elles sont alors prises en charge par la machinerie de l'autophagie. La protéine IRGM est à nouveau directement impliquée puisque son niveau d'expression est directement corrélé à la capacité des cellules épithéliales à contrôler la multiplication des bactéries intracellulaires [5]. La protéine ATGl6Ll intervient également, car si l'on diminue son expression dans des cellules épithéliales intestinales par l'utilisation d'ARN interférents, Salmonella typhimurium n'est plus contrôlée par l'autophagie [6].

Ces différents exemples montrent l'importance du rôle qu'exerce l'autophagie dans l'immunité innée de l'hôte vis-àvis d'une infection bactérienne. De plus, la dégradation des pathogènes dans l'autophagolysosome permet d'alerter l'immunité adaptative, via la présentation d'antigènes par les molécules du $\mathrm{CMH}$ (complexe majeur d'histocompatibilité) de classe II.

\section{Mise en évidence}

de polymorphismes dans des gènes clés de l'autophagie chez les patients atteints de maladie de Crohn

La maladie de Crohn (MC) est une maladie inflammatoire chronique du tube digestif qui affecte environ 1 personne sur 1000 dans les pays occidentaux, soit environ 1 million de personnes dans le monde [16]. Un certain nombre d'arguments épidémiologiques, cliniques et expérimentaux plaident en faveur du rôle de la flore digestive dans l'étiologie de la MC : la dérivation du contenu luminal entraîne une rémission de la maladie tant que la muqueuse n'est pas au contact du flux fécal, et l'administration d'antibiotiques permet d'améliorer les signes cliniques de la maladie.
Les avancées en génétique humaine ont mis en évidence l'importance de l'immunité innée et la relation d'équilibre entre microbiome intestinal et muqueuse intestinale de l'hôte dans la pathogenèse de la MC. Des mutations dans un premier gène de susceptibilité avaient été mises en évidence en 2001 chez des patients présentant une atteinte iléale de la $M C$ [7]. II s'agit du gène nod2 codant un récepteur intracytoplasmique capable de reconnaître le muramyl dipeptide bactérien et d'enclencher une réponse innée dans la cellule hôte. L'identification d'un tel gène indiquait déjà une piste infectieuse, via des bactéries invasives, dans la pathogénie de la MC. Récemment, des études indépendantes d'association à l'échelle du génome ont identifié des variants du gène ATGI6LI et d'une délétion en amont du gène IRGM (interferon-inducible guanosine triphosphatase), comme marqueurs de prédisposition génétique $[6,8-10]$. Les protéines ATG16Ll et IRGM étant indispensables à l'induction de l'autophagie, cela renforce I'hypothèse du rôle de bactéries invasives et de l'absence du contrôle de leur persistance et/ou de leur multiplication intracellulaires dans la MC.

\section{Implications de bactéries}

invasives dans la maladie de Crohn

Dès les premières études bactériologiques, les similitudes entre MC et certaines formes d'entérocolites infectieuses de type shigellose, salmonellose ou yersiniose ont permis d'évoquer l'implication de bactéries invasives à tropisme intestinal dans l'étiologie de la MC. Les bactéries suspectées sont Mycobacterium avium subspecies paratuberculosis (MAP) et des souches de Escherichia coli adhérentes et invasives (AIEC), deux bactéries à tropisme intracellulaire et capables de survivre et de se multiplier dans les cellules épithéliales et/ou les macrophages de l'hôte. Si les résultats des analyses recherchant la présence de MAP chez les patients atteints de MC sont très contradictoires, les arguments s'accumulent en faveur de l'implication 
A

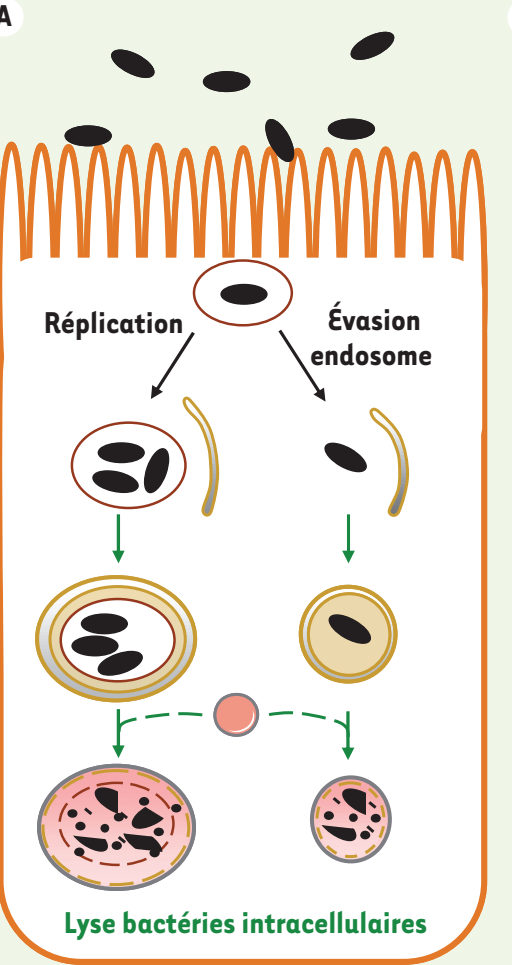

des AIEC [11]. La prévalence de souches AIEC au niveau iléal est de $36,4 \%$ chez les patients atteints de MC contre seulement $6,2 \%$ chez les sujets qui ne sont pas atteints de pathologies inflammatoires intestinales [12]. La forte colonisation par des bactéries AIEC est due à l'expression anormale de molécules CEACAM6 (human carcino-embryonic antigen cell adhesion molecule) au niveau iléal chez les patients [13]. L'expression accrue de CEACAM6, récepteur impliqué dans l'adhésion des AIEC, serait consécutive à l'infection, ces bactéries étant capables d'induire leur propre colonisation.

Les connaissances actuelles ne permettant pas d'établir si les bactéries AIEC peuvent moduler le processus d'autophagie, mais il est probable qu'une altération de ce mécanisme chez des patients atteints de $M C$ contribuerait à la mise en place de conditions facilitant la persistance et la multiplication de ces bactéries pathogènes invasives (Figure 2). $\diamond$
B

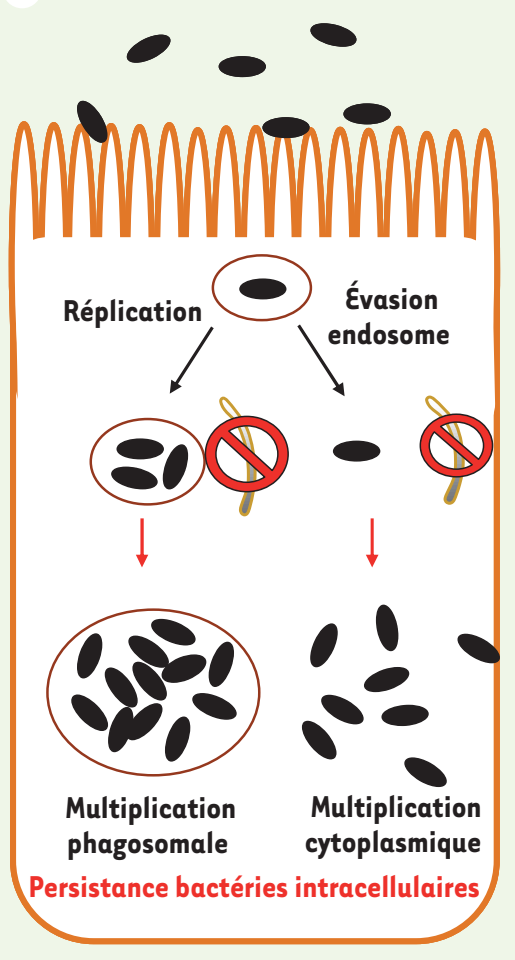

Impaired autophagy in Crohn's disease patients: an opened gate to invasive bacteria?

\section{REMERCIEMENTS}

Nous remercions pour leur soutien l'Association François Aupetit dédiée à l'amélioration des connaissances sur les maladies inflammatoires chroniques intestinales (maladie de Crohn et rectocolite hémorragique), l'Institut de Recherche des Maladies de l'Appareil Digestif (Laboratoire AstraZeneca), l'institut National de la Recherche Agronomique et le Ministère de l'Enseignement Supérieur et de la Recherche.

\section{RéFÉRENCES}

1. Xie Z, Klionsky DJ. Autophagosome formation: core machinery and adaptations. Nat Cell Biol 2007 ; 9: 1102-9.

2. Nakagawa I, Amano A, Mizushima N, et al. Autophagy defends cells against invading group A Streptococcus. Science 2004 ; 306 : 1037-40.

3. Gutierrez MG, Master SS, Singh SB, et al. Autophagy is a defense mechanism inhibiting BCG and Mycobacterium tuberculosis survival in infected macrophages. Cell 2004 ; 119: 753-66.
Figure 2. Autophagie et contrôle de bactéries intracellulaires. A. L'autophagie contribue à l'immunité innée en dégradant des bactéries soit cytoplasmiques soit intraphagosomales qui sont capables de résister à la dégradation par le trafic endocytaire. $B$. Une altération du mécanisme de l'autophagie chez des patients génétiquement prédisposés, avec mutation ATG16L1300A et/ou délétion en amont du gène IRGM, pourrait conduire à un défaut de prise en charge des bactéries intracellulaires, favorisant ainsi leur persistance.

4. Singh SB, Davis AS, Taylor GA, Deretic V. Human IRGM induces autophagy to eliminate intracellular mycobacteria. Science 2006 ; 313 : 1438-41.

5. McCarroll SA, Huett A, Kuballa P, et al. Deletion polymorphism upstream of IRGM associated with altered IRGM expression and Crohn's disease. Nat Genet 2008; 40: 1107-12.

6. Rioux JD, Xavier RJ, Taylor KD, et al. Genome-wide association study identifies new susceptibility loci for Crohn disease and implicates autophagy in disease pathogenesis. Nat Genet 2007 ; 39 : 596-604.

7. Hugot JP, Chamaillard M, Zouali H, et al. Association of NOD2 leucine-rich repeat variants with susceptibility to Crohn's disease. Nature 2001 ; 411 : 599-603.

8. Wellcome. Genome-wide association study of 14,000 cases of seven common diseases and 3,000 shared controls. Nature 2007 ; 447 : 661-78.

9. Parkes M, Barrett JC, Prescott NJ, et al. Sequence variants in the autophagy gene IRGM and multiple other replicating loci contribute to Crohn's disease susceptibility. Nat Genet $2007 ; 39: 830-2$.

10. Hampe J, Franke A, Rosenstiel P, et al. A genome-wide association scan of nonsynonymous SNPs identifies a susceptibility variant for Crohn disease in ATG16L1. Nat Genet 2007 ; 39 : 207-11.

11. Sartor RB. Microbial influences in inflammatory bowel diseases. Gastroenterology 2008 ; 134 : 577-94.

12. Darfeuille-Michaud A, Boudeau J, Bulois P, et al. High prevalence of adherent-invasive Escherichia coli associated with ileal mucosa in Crohn's disease. Gastroenterology 2004 ; 127 : 412-21.

13. Barnich N, Carvalho FA, Glasser AL, et al. CEACAM6 acts as a receptor for adherent-invasive $\varepsilon$. coli, supporting ileal mucosa colonization in Crohn disease. J Clin Invest 2007 ; 117: 1566-74.

14. Pattingre $S$, Codogno P. L'autophagie et le dernier soupir de la cellule. Med Sci (Paris) 2007 ; $23:$ 696-8.

15. Codogno P. Les gènes ATG et la macro-autophagie. Med Sci (Paris) $2004 ; 20: 734-6$.

16. Peyrin-Biroulet L, Parmentier-Decrucq $\varepsilon$, Branche J, Desreumaux P. L'IL-23R, un nouveau gène de susceptibilité dans les maladies inflammatoires chroniques intestinales. Med Sci (Paris) 2007 ; $23: 250-2$.

17. Codogno P. L'autophagie, dégrader plus pour vivre plus... mais attention! Med Sci (Paris) 2009 ; 25 : 323-4.

18. Puyal J, Ginet V, Vaslin A, et al. Les deux visages de l'autophagie dans le système nerveux. Med Sci (Paris) $2009 ; 25: 383-90$. 